\title{
La evaluación formativa, un camino por recorrer en la práctica docente
}

\author{
Formative Assessment: a Teaching-Practice \\ Road to Go.
}

Mtra. Blanca Ponce Cortés*

*Profesora de Carrera de la Escuela Nacional de Enfermería y Obstetricia

\section{Resumen}

En el presente articulo se presentan los elementos teóricos que guiaron el subproyecto PAPIME No. 223003“Evaluación de la practica docente de los profesores de la Escuela Nacional de Enfermería y Obstetricia. Un enfoque formativo"

En el primer apartado se abordan dos enfoques de la evaluación: el sumativo (cuantitativo) y el formativo (cualitativo) para distinguir la importancia de la elección de uno u otro. El primero esta orientado a medir los resultados, el segundo, esta centrado en los procesos, buscando proporcionar información sobre actividades que se desarrollan a fin de mejorar el desempeño a evaluar.

En el segundo apartado se destaca la importancia de evaluar la práctica docente de los profesores a través no sólo

Theoretical elements which guided the subproject "PapimeTeaching Evaluation of the Escuela Nacional de Enfermeria y Obstetricia are presented in the following paper.

In the first paragraph, two focuses of evaluation are considered: quantitative and qualitative to distinguish the choice of importance between one and the other. The first oriented to measure the results of the second, it is focused in the processes, looking to provide information about the activities that are developed to enhance the performance that is being evaluated.

In the second paragraph, the importance of evaluating teaching practice, not only through the students, irreplaceable de los estudiantes, informantes irremplazables, sino también de la mirada de los profesores sobre su práctica, por la importancia de conocer sus pensamientos al respecto, ya que consideramos que constituyen uno de los fundamentos para actuar sobre el currículo y sobre la mejora de sus prácticas.

Concluimos considerando que este camino por recorrer hacia la evaluación formativa es un terrero fértil para la sensibilidad y la exploración de nuevas y efectivas formas de evaluación y estrategias de formación docente que conduzcan a la mejora del proceso formativo de los alumnos.

Palabras clave evaluación formativa, evaluación sumativa, práctica docente.

\section{Abstract}

informer, but also through the point of view of the teachers about their own teaching and knowing about their thoughts and ideas is highlighted since we consider that they constitute one of the fundamentals to go about their curricula and the enhancement of their teaching practice.

We can conclude that the road to formative evaluation is a fertile land for the sensitivity and exploration of the new and effective forms of the evaluation and strategies of teacher training which will lead to enhance the forming process of the students

Key words Qualitative evaluation, quantitative evaluation, teaching practice 


\section{INTRODUCCIÓN}

El presente escrito se circunscribe en el Anteproyecto "Evaluación de la práctica docente de los profesores de la Escuela Nacional de Enfermería y Obstetricia. Un enfoque formativo" ubicado en el Programa de Apoyo a Proyectos Institucionales para el Mejoramiento de la Enseñanza (PAPIME), aprobado en 2003 y concluido en 2007. El grupo de trabajo estuvo integrado por seis profesores y un asesor externo.

Este Subproyecto surgió a partir de la intención de darle continuidad a los procesos de evaluación de la práctica docente llevados a cabo en la escuela, para lo cual, el grupo de trabajo tuvo como primera actividad su revisión y análisis el producto de la reflexión sobre dichos procesos fue el coincidir en la necesidad de resignificar la función de la evaluación y sus procesos y a partir de esta resignificación reorientarlo en dos puntos esenciales: a) introducir una metodología participativa y b) vincular los resultados con estrategias de formación docente dirigidas a la mejora de la práctica.

Esta búsqueda por replantear el concepto mismo de la evaluación implicó la confrontación de nuestros esquemas referenciales sobre la misma, que si bien, estaban planteados algunos aspectos en el subproyecto, en el momento de diseñar la construcción del instrumento de evaluación había ciertas divergencias en algunos conceptos y, después de la revisión bibliográfica sobre el tema y de la lectura de algunas experiencias de evaluación docente en otras instituciones y de largas discusiones, nos preocupó lo controvertido y complejo de esta temática a la que nos teníamos que enfrentar. El debate por lo complejo de las posturas teóricometo dológicas existentes y por las variables personales y contextuales que intervienen en la práctica docente, así como la dificultad que implica desterrar las resistencias y temores de los profesores ante la evaluación, generados por prácticas evaluativas tradicionales donde los docentes, las más de las veces, son vistos como objetos.

El reto a seguir es cambiar la visión de la evaluación como una amenaza y la de los evaluadores como jueces que sancionan, situación que hace necesario trabajar en el desarrollo de una cultura de la evaluación con los docentes, alumnos y autoridades que en conjunto generen un ambiente propicio para la misma, lo que favorecería la sensibilidad y el convencimiento de todos los actores respecto a la conveniencia de que esta práctica genere en ellos la necesidad de solicitarla y de participar en el proceso, así como, a partir de los resultados obtenidos, vincularlos con estrategias de formación docente que coadyuven a mejo- rar significativamente su práctica, pues es a través de ella que los proyectos educativos se hacen realidad. De aquí el enfoque formativo del anteproyecto cuyo objetivo central es comprender para mejorar la práctica docente de los profesores.

En este artículo se presentan algunos elementos teóricos sobre la evaluación formativa por lo que nos pronunciamos y que constituyeron el eje sobre el cual giraron las acciones emprendidas en el subproyecto quedando para un próximo trabajo la presentación de la metodología seguida y los resultados obtenidos. Asimismo, se enfatiza en la necesidad de incorporar la mirada del propio docente sobre su práctica la que, aunada a las opiniones de los alumnos, enriquecerá y dará mayor sentido a las acciones encaminadas al mejoramiento de la docencia

\section{ENFOQUES DE LA EVALUACIÓN}

Hoy en día la naturaleza de las instituciones universitarias reclaman la existencia de procesos internos y externos de evaluación como procedimientos necesarios para garantizar la pertinencia, eficacia y eficiencia de las mismas.

En este contexto surgen distintas instancias evaluadoras como los Comités Interinstitucionales para la Evaluación de la Educación Superior (CIIES), El Consejo Mexicano de Acreditación y Certificación de Enfermería, A.C. (COMACE) entre otros, con el propósito de evaluar las instituciones, los planes y programas de estudio que más tarde se ampliarían a la evaluación curricular, y en las últimas décadas, la evaluación de los docentes ha sido una preocupación importante de las instituciones de educación superior.

La evaluación es una dimensión fundamental del campo educativo y requiere mayor atención si asumimos que en buena medida de ella depende tanto la actividad de los profesores como el aprendizaje de los alumnos, la gestión de las autoridades y el sistema en su conjunto. ${ }^{1}$

Para este autor, en el campo de la evaluación académica lo más frecuente es encontrar dos posiciones respecto a la función que debe cumplir: formativa o sumativa. La formativa está centrada en los procesos, buscando proporcionar información respecto a las actividades que se desarrollan, a fin de retroalimentar el desempeño que se está evaluando. El énfasis de esta evaluación se pone en el desarrollo, en el mejoramiento, en el proceso, considerado individualmente, dándole un sentido esencialmente constructivo y propositivo, en la que todos los involucrados tengan la oportunidad de participar y, como consecuencia, mejorar su actuación. 
Al respecto Santos Guerra ${ }^{2}$ plantea que si bien, la evaluación de resultados, el establecimiento de juicios de valor y la toma de decisiones son algunas de las finalidades de la evaluación, considera que estos enfoques dejan de lado algunos aspectos que le parecen centrales y que destaca a partir de su concepción de la evaluación como un proceso de diálogo, comprensión y mejora, señalando las características que a su juicio debe tener la evaluación. Estos rasgos son:

- Independiente, que no esté sometida, sojuzgada, vendida o simplemente alquilada al poder, el dinero o la tecnología.

- Cualitativa, y no meramente cuantificable, pues la reducción a números suele simplificar y desvirtuar la parte más sustantiva de los mismos.

- Práctica y no meramente especulativa, pues tiene como finalidad la mejora de los programas a través de su comprensión, a través del conocimiento de su naturaleza, funcionamiento y resultados.

- Democrática y no autocrática en tanto que la evaluación se pone al servicio de los usuarios, no del poder, porque cuando se utiliza torcidamente, produce efectos negativos.

- Procesual, no meramente final, porque es durante el mismo cuando se puede conocer lo que sucede en él y durante su desarrollo se puede modificar y mejorar.

- Participativa, no mecanicista, que de voz a los participantes, son ellos los que emiten su valoración sobre su desempeño, aunque no sea la única voz y la única perspectiva que se tiene en cuenta.

- Colegiada, no individualista, porque la evaluación realizada por un equipo goza del aval del contraste, de la pluralidad de enfoques, de una mayor garantía de rigor, de una diversificación estratégica de acceso y actuación.

- Externa, aunque de iniciativa interna, donde los propios participantes y usuarios la demanden y para realizarla requieren de la colaboración del exterior.

Parafraseando a este mismo autor, la conexión entre las tres dimensiones de la evaluación que plantea, diálogo, comprensión y mejora, potencia a cada una en tanto que forman parte de un mismo proceso pues, mientras se realiza el diálogo entre evaluadores y evaluados y porque se realiza, se produce la comprensión. Cuando se produce la comprensión es más fácil y enriquecedor el diálogo y el diálogo fecundo es una parte de la mejora de la práctica. Esto no significa que en algún momento no existan conflictos entre el evaluador y los evaluados pues éstos son inherentes en la vida institucional y en las experiencias cotidianas, silenciarlos o negarlos impediría aprovecharlos como fuente de conocimiento.

En cuanto a la evaluación sumativa, se trata fundamentalmente de una "valoración del producto" o del resultado. Lo importante es asignarle un valor numérico a lo que se está juzgando, mismo que servirá para un proceso de toma de decisiones: calificación de un alumno, otorgar incentivos a los profesores, la renovación de un contrato, el ingreso a la movilidad laboral, etc.

Esta concepción de la evaluación que ha dominado en el campo educativo, ha generado tanto en profesores como en alumnos una predisposición ante la misma, cuestionando la validez y confiabilidad del instrumento, la representatividad de lo que se quiere evaluar, el uso de los resultados, la forma en que se aplica y dudando también de quien lo aplica. $^{3}$

Ambos enfoques de la evaluación, la formativa y sumativa, se ubican en dos grandes paradigmas de investigación (Bryman, 1984). ${ }^{4}$ Por un lado el llamado positivismo racionalista, derivado de las ciencias naturales y exactas. Por otro, el interpretativo, también llamado fenomenológico o hermenéutico, derivado de las ciencias humanas. Estos paradigmas tienen supuestos diferentes y dan lugar a metodologías de investigación diferentes. En el primer paradigma, positivista, se desarrollan las metodologías cuantitativas, experimentales y las hipotético deductivas. En el segundo, el subjetivista, las metodologías cualitativas, interpretativas y empírico deductivas.

La disputa entre los dos enfoques ha dado paso a la posibilidad de una complementariedad de ambos dado que se advierte que ninguno de los dos puede agotar el análisis y la representación de la realidad (Bryman, 1984), Ninguna de las dos aproximaciones por sí solas puede ofrecer un panorama completo de lo que se evalúa o de lo que se investiga, como señala Ericksson (1989), lo más sensato es decidir qué tipo de cosas tiene sentido narrarlas, observarlas o grabarlas, y cuáles otras es preferible contarlas o calificarlas. ${ }^{5}$

El establecer las bondades académicas de la evaluación cualitativa o formativa no ha sido ni es tarea fácil, pues está muy introyectado en el ámbito académico el criterio de "objetividad", aquel que se sustenta en la teoría de la medición, cuya consigna explícita consiste en sumar, promediar y asignar calificaciones en exámenes parciales y finales a través de incipientes o bien sofisticados tratamientos estadísticos, en el caso de los alumnos. 
Siguiendo a Juan Manuel Álvarez ${ }^{6}$, en estricto sentido, toda la evaluación es cualitativa, y lo es si tenemos en cuenta que la llamada, por contraposición, cuantitativa, comienza precisamente a partir de los datos cuantificados y justo donde los números no llegan, comienza la evaluación. Evaluar no es otra cosa que valorar, enjuiciar o, mirando el resultado, evaluar no es ni más ni menos que la emisión de un juicio sobre la base de la información e interpretación de la misma obtenida por distintos medios, el estadístico si procede, pero ello no implica supeditarse a la frialdad del dato.

Centrar la mirada hacia la evaluación formativa o cualitativa, por la que nos pronunciamos, la entendemos no como una actitud de enjuiciamiento para calificar o descalificar el desempeño de un docente. La evaluación docente la concebimos como un proceso de conocimiento y reflexión, donde los profesores se convierten en elementos centrales de la misma, como un proceso movilizador en tanto abra un espacio de cuestionamiento de lo que se hace y por qué razones, que permita a los docentes tomar conciencia de los aspectos que necesita cambiar para mejorar su práctica docente.

En la actualidad diversos estudios sobre la evaluación docente participativa han demostrado que diseñar un instrumento que recupere la postura de profesores y estudiantes e incluso la visión institucional, es un buen paso para la sensibilización de docentes y alumnos para cambiar sus maneras de entender las formas y usos de la evaluación docente. También se ha podido demostrar que a través de la evaluación formativa, el docente toma conciencia, reflexiona sobre su actuación en el aula, es decir, se autoevalúa, transforma y logra mejorar su docencia y objetivos ${ }^{7}$.

\section{¿Por qué y para qué evaluar la práctica docente?}

Hoy más que nunca los planes de estudio no pueden competir con el avance diario de los conocimientos, la evolución tecnológica y las transformaciones en los procesos laborales y, mientras más acelerados son los cambios, más importante es el desarrollo de habilidades intelectuales, actitudinales y sociales por parte de los alumnos para su aprendizaje permanente.

Entre los objetivos actuales de la educación destaca, la autonomía para aprender que es susceptible de lograrse por las posibilidades que tienen los alumnos de aprender

\section{"Evaluación de la docencia, ese espejo que refleja nuestra} imagen a partir de la mirada del otroy de nosotros mismos; habilidades, actitudes,formas de ser y de comunicar, imagen que por momentos nos conduce a la autocomplacencia, imagen que en ocasiones molesta e incomoda, pero al fin y al cabo, imagen necesaria. Evaluación de la docencia, la puerta que nos posibilita un reencuentro con el análisis y lareflexión de la práctica docente" 
de manera independiente a partir de sus propias capacidades y estrategias sin que esto excluya la presencia del profesor pero sin la dependencia extrema. Sin embargo, la docencia actual, a pesar de los avances en la investigación educativa y de los programas de formación de profesores de los últimos años, se ha convertido frecuentemente en una actividad mecánica, improvisada y fría en donde el profesor, las más de las veces, asume el papel protagónico y el alumno el de escucha obediente, desapareciendo así la opción primordial del diálogo en el acto de enseñar y aprender.

Para Raquel Glazman, la docencia es una tarea intencionada que implica claridad de finalidades; dominio del contenido por parte del maestro; uso apropiado de métodos didácticos, y conocimiento específico sobre las condiciones del estudiante, como saber, capacidad, hábitos, actitudes y recursos. Es un proceso de comunicación matizado por las intenciones de la institución, el maestro y el estudiante, pero que rebasa la comunicación entendida en un sentido restringido de transmisión de mensajes, y que requiere tratamientos detallados por parte de quien enseña para promover habilidades, capacidades, destrezas y conocimientos.

Bajo esta concepción de docencia y en el contexto del currículum formal o institucional en el que se inscriben diversos aspectos: necesidades de la profesión, perfil de egreso de los alumnos, programas de las asignaturas entre otros, se derivaron las características del docente, referente básico de la evaluación realizada.

En esta perspectiva de la forma en que los docentes practican el currículum formal se planteó la evaluación de la práctica docente de los profesores de la Escuela Nacional de Enfermería y Obstetricia desde la opinión de los alumnos, lo que no significa, de ninguna manera, que se consideren las encuestas hechas como el único medio para obtener información, pensamos que el proceso de evaluación deberá incorporar otros medios e informantes como son: la autoevaluación, la observación de pares, el análisis conjunto entre evaluadores y docentes, el portsafolio, entre otros, para ir desarrollando propuestas de mejora.

Como señalan algunos autores (Tirado, 1990, De la Orden, 1990, Zabalza, 1990, entre otros) $)^{9}$, es cierto que las opiniones de los alumnos pueden estar influidas por diversos factores (alguna experiencia positiva o negativa, mayor o menor empatía con el profesor, resultados obtenidos en el curso) sin embargo el alumno es una excelente fuente de información, son ellos quienes pasan el mayor tiempo con los profesores, quienes perciben directamente la intención positiva o negativa del maestro, las habilidades en la comunicación, la presencia de planeación del curso, la coherencia y justicia en la evaluación. Son los usuarios y eso los convierte en los informantes de primera mano, a todas luces irremplazables.

Si bien hemos obtenido información valiosa por parte de los alumnos y a partir de la cual se han generado algunas acciones de intervención, somos concientes de que esta miradarespecto a lo que hacen y no hacen los profesores no es suficiente para una evaluación más amplia de su práctica, faltaría indagar sobre el pensamiento de los docentes respecto a la misma práctica, lo que permitiría explicar por qué los profesores hacen o dejan de hacer ciertas cosas establecidas en el currículum formal en la cotidianidad de sus actividades

La necesidad de considerar lo que piensa el profesor (teorías implícitas, representaciones, imágenes, suposiciones, intenciones, creencias, actitudes, intereses y valores, etc.) radica en que es uno de los fundamentos para actuar sobre el currículum y sobre la mejora de las prácticas ${ }^{10}$. Coincidimos con Gimeno Sacristán cuando señala que, cualquier idea importante, cualquier nuevo planteamiento para la práctica educativa pasará a ésta de forma efectiva, en la medida que impregne los pensamientos y la forma de actuar de los profesores. La mentalidad y las prácticas de los profesores son los caminos a través de los que las ideas transforman la realidad.

Lo anterior nos remite a la necesidad de indagar sobre la forma en que los profesores interpretan, adaptan y trabajan el currículum formal creando una práctica propia que puede parecerse a lo diseñado previamente pero que también puede estar muy distanciado y que potencialmente es más importante que el formal y que constituye el currículum real. ${ }^{11}$

Por lo antes expuesto podemos asumir que la evaluación de la docencia es un tema de interés relevante y actual de la educación superior, considerada como una actividad valorativa e investigadora, facilitadora del cambio y desarrollo educativo. Su riqueza estriba en su capacidad para ofrecer datos e interpretaciones significativas que permitan entender y valorar el desarrollo de los procesos y de los resultados obtenidos, sean éstos esperados o no, y a partir de ellos buscar la mejora. En suma, evaluar es más que calificar; significa enjuiciar, tomar decisiones sobre nuevas acciones a emprender y, en definitiva, transformar para mejorar.

\section{REFLEXIONES FINALES}

Si bien la práctica docente constituye el espacio donde se generan los verdaderos cambios, es preciso reconocer sus determinaciones individuales, institucionales y sociales para percatarse de la complejidad que entraña su evaluación. 
El profesor, como sujeto social tiene introyectada una visión de mundo que se expresa en un esquema conceptual con el que piensa y actúa, el cual puede coincidir o discrepar con los planteamientos del currículum formal; asimismo su situación personal, edad, intereses, motivaciones, expectativas, creencias, actitudes, valores, formación profesional, etc., son factores que le posibilitarán asumir, rechazar o simular la propuesta.

Esta práctica en tanto se da en un espacio institucional también está condicionada por las características de la misma, normas explícitas e implícitas, relación entre autoridades y profesores, organización y relaciones entre profesores, dinámica académica, organización administrativa, etc., condiciones que pueden dinamizar o paralizar los cambios deseados.

Con el título del trabajo, "La evaluación formativa un camino por recorrer en la práctica docente", queremos destacar que romper con patrones tan arraigados como es la actitud de rechazo y suspicacia que normalmente se tiene hacia la evaluación, nos hace pensar que estos cambios no se darán de la noche a la mañana sino que requerirán de procesos de mediano y largo plazo y de condiciones de apoyo institucional orientados hacia la intencionalidad pedagógica de la evaluación docente y, como resultado de ésta, experimentar propuestas de intervención educativa vinculadas con los resultados de la evaluación docente desde la mirada de los estudiantes sin dejar de considerar la otra mirada, la de los profesores, con el propósito de encontrar nuevos caminos que permitan mejorar su práctica, lo que repercutirá en una formación académica de mayor calidad.

Reconociendo las características de la evaluación planteadas por Santos Guerra, consideramos que la práctica docente tendría que ir precisamente en el mismo sentido, lo que implicaría un nuevo estilo de trabajo académico, el colegiado, donde los coordinadores, con los docentes establezcan y apoyen las líneas de acción necesarias que contribuyan a la mejor formación de los estudiantes, buscando plenamente a través de este trabajo colectivo y participativo el consenso de los actores en torno a la intencionalidad de las mismas.

Concluimos considerando que este camino por recorrer hacia la evaluación formativa es un terreno fértil para la sensibilidad y la exploración de nuevas y efectivas estrategias de evaluación y de formación docente que conduzcan a una mejor formación de los alumnos, razón de ser de toda institución educativa.

\section{REFERENCIAS BIBLIOGRÁFICAS}

1 Canales-Alejandro, Evaluación educativa. La oportunidad y el desafío, en la Revista Reencuentro. Análisis de problemas universitarios, Evaluación cualitativa en la enseñanza universitaria, Abril, México 2007, p.41.

2 Santos-G; Miguel Ángel, La evaluación: un proceso de diálogo, comprensión y mejora, Ediciones Aljibe, $2^{a}$. Ed. Málaga, 1995, p.p. 35-36

3 Loredo-E; Javier, Presentación, En: Evaluación de la práctica docente en educación superior (Coordinador) Ed. Porrua, Universidad Anáhuac, México, 2000 p.p290

4 Álvarez; Juan Manuel, Valor social y académico de la evaluación. Revista Alternativas, Año III, No. 5. Buenos Aires, 1989. p. 1

5 Cruz-R; Isabel, Et. Al. La evaluación formativa: Estrategia para promover el cambio y mejora en la docencia en M. Rueda y F. Díaz Barriga (coordinadores) Evaluación de la docencia. Perspectivas actuales, Ed. Paidos Educador, México, 2000. p. 149

6 Glazman-N; Raquel, El vínculo docencia-investigación en la universidad pública, en P. Morán (Coordinador) Docencia e investigación en el aula. Una relación imprescindible, Pensamiento Universitario, Tercera Época, 92, CESU, UNAM p.78.

7 Loredo-E; Javier, Presentación, En: Evaluación de la práctica docente en educación superior (Coordinador) Ed. Porrua, Universidad Anáhuac, México, 2000, p. x

8 Monroy-F: Miguel, Evaluación de la práctica educativa a través de la reflexión del pensamiento didáctico docente, en M. Rueda y F. Díaz Barriga (coordinadores) Evaluación de la docencia. Perspectivas actuales, Ed. Paidos Educador, México, 2000. p. 283

9 Figueroa-R; Alma Elena, Una alternativa para la evaluación de la enseñanza en educación superior desde la perspectiva de los profesores, en M. Rueda y F. Díaz Barriga (coordinadores) Evaluación de la docencia. Perspectivas actuales, Ed. Paidós Educador, México, 2000. p.261

\section{DIRECCION PARA CORRESPONDENCIA}

Mtra. Blanca Ponce Cortés: blancajulia01@hotmail.com 